\section{FciDioc \\ Quality of Life of Elderly and Obese Animal Models with Severe Knee Osteoarthritis after a Single Injection of Mesenchymal Stem Cells \\ International Journal of Stem Cell Research and Transplantation (IJST) ISSN:2328-3548}

Research Article

Phermthai $\mathrm{T}^{1^{*}}$, Pokathikorn $\mathrm{P}^{1}$, Thongbopit $\mathrm{S}^{1}$, Chareancholvanich $\mathrm{K}^{2}$, Parnpai $\mathrm{R}^{3}$, Julavijitphong $\mathrm{S}^{1}$, Tungprasertpol $\mathrm{K}^{1}$

${ }^{1}$ Stem Cell Research and Development Unit, Department of Obstetrics \& Gynecology, Faculty of Medicine Siriraj Hospital, Mahidol University, Bangkok, Thailand.

${ }^{2}$ Department of Orthopedic Surgery, Faculty of Medicine Siriraj Hospital, Mahidol University, Bangkok, Thailand

${ }^{3}$ Embryo Technology and Stem Cell Research Center and School of Biotechnology, Suranaree University of Technology, NakhonRatchasima, Thailand.

\title{
Abstract
}

Knee osteoarthritis $(\mathrm{OA})$ is a hazardous degenerative disease causing daily disability and great suffering for the world's population. The goals of OA treatment are alleviation of pain and recovery of function status. So far, several conservative care options (non-surgical) are available, but only provide temporary relief for the clinical symptoms of severe OA. When joint replacement surgery is required, older people tend to avoid surgical treatment, due to the risk of serious side effects. That causes great suffering and loss of quality of life. Currently, mesenchymal stem cells (MSC) therapy has been discussed as a treatment of OA. Here, we investigated a feasibility of MSC therapy as an option of a simple treatment for OA in the aged population. We transplanted an intra-articular injection of human MSC into the knee joint of an aged animal model, which carry natural severe OA, and monitored for pain, knee function. Their quality of life was evaluated via ARomDe scores. Our results demonstrated that those aged animals have good recovery of the knee and leg functions. Their daily activities and behavior well-being (walking, grooming, and social interaction) had increased. We conclude that single MSC injections have a good effect and provide a better quality of life in aged animal model with severe OA knee.

Keywords: Animal Model; Knee; Mesenchymal Stem Cells; Osteoarthritis; Quality of Life.

\section{Introduction}

Knee osteoarthritis (OA) is a chronic disorder and degenerative disease of the connective tissue, which is a cause of disability in both genders of the elderly and obese population. It is characterized by progressive damage and loss of articular cartilage resulting in pain and disability in OA patients $[1,2]$. The patients greatly suffered in their activities of daily living (walking, stair climber) and declined in the quality of life, social interaction, mental efficiency and sleep quality [3]. The high prevalence of OA makes it recognizable as a public health hazards issue. In US, about $37 \%$ of the populations over 60 years of age are diagnosed with OA [2]. It is estimated that the incidence of disease will increase to $40 \%$ of the world population in 2025 , due to aging and obesity [2, 4].
The progressive damage of $\mathrm{OA}$ is classified into mild, moderate and severe stages depending on clinical symptoms and disruption of articular cartilage and knee joint function [5]. In mild and moderate $\mathrm{OA}$, the clinical symptoms can be relieved by conservative treatments including physical therapy, dietary supplements; pharmacological therapy with steroids, NSAIDs, analgesics and even viscosupplementation with an injection of hyaluronic acid [6]. In severe knee $\mathrm{OA}$, conservative treatment only provides temporary relief of clinical symptoms. Total knee replacement is required [7]. However, no treatment has been shown as ideal, so far, regarding their side effects. In the case of older adult patients with severe OA, total knee surgery has been considered to have a high risk of mortality, complications during the operation (such as side effects from anesthesia) or in postoperative stage and lengthy re-

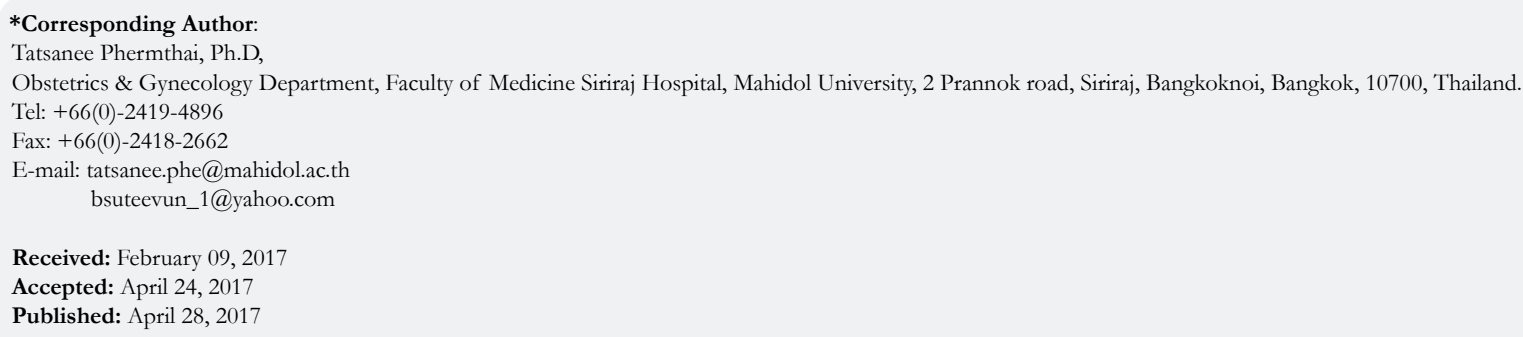

Copyright: Phermthai $\mathbf{T}^{\circ}$ 2017. This is an open-access article distributed under the terms of the Creative Commons Attribution License, which permits unrestricted use, distribution and reproduction in any medium, provided the original author and source are credited. 
covery time [8-11].

In this decade, an alternative medicine for knee OA was highlighted as mesenchymal stem cell (MSC) therapy [12-14]. The advantage of stem cell therapy over traditional approaches is the restoration of cartilage function in the knee. MSC promotes the migration of endogenous repairing cells to injury sites and the self-healing process of the knee joint cartilage, resulting in relief of OA symptoms and an improved patient functional status [14-18]. Whether or not regeneration of knee cartilage has been achieve by stem cell therapy in elderly people, a better quality of life; reduction of pain, a disability of daily living activities and mental suffering, is worth the effort to try.

A number of recent reports have revealed scientific evidence and therapeutic success with MSC therapy for OA. Soler et al., 2016 [14] treated OA patient with an intra-articular injection of MSC and reported a long-lasting amelioration of pain and signs of cartilage repair. Broeckx et al., 2014 [19] presented the success of OA treatment in equine with a transplantation of equine MSC. Tsai et al., 2014 [20] demonstrated that a single intra-articular injection of porcine adipose-derived stem cells into the $\mathrm{OA}$ joint of dogs could improve mobility, activity and lessened pain. Their study exhibited that xenotransplantation of MSC for the treatment of $\mathrm{OA}$ is feasible. Guinea pig is an interesting animal model for OA disease; due to spontaneous OA in guinea pig is naturally occurring in this slowly progressive disease. The histological lesions observed in guinea pig are similar to those of human OA [21]. In 2012, Sato et al., [22] revealed the evidence of cartilage regeneration by human MSC transplantation in the OA knee joint of guinea pig.

The aim of our study was to monitor the effect of OA treatment with simple MSC therapy to the quality of life of an aged animal model with spontaneous severe knee OA. Single intra-articular injection of human MSC was applied to the degenerated knee joint of guinea pig and evaluated for a specific period of time to achieve recovery and increased quality of life by following the "ARomDe" score. We were setting up for the first time for the "ARomDe" scoring system to evaluate the progression of OA treatment in an aged animal model with severe OA. This assessment can measure in three dimensions, including assessment of pain, function recovery and emotional behavior.

\section{Materials and Methods}

\section{Animals}

Twenty male Dunkin Hartley guinea pigs (National Laboratory Animal Center, Mahidol University, Thailand) at two months of age weighing about $500 \mathrm{~g}$ each were maintained and fed individually in ventilated cages on a $12 \mathrm{~h}$ light/dark cycle with the temperature at $25^{\circ} \mathrm{C}$ and with abundant water and food. The animals were cared for and observed during a walking test every two weeks to find out the optimal period of time to demonstrate uncomfortable walk behavior in guinea pig model. Animal model, aged 2.5 years, weighing over $850 \mathrm{~g}$, was used in the experiment. All animals were handled according to the regulations of the Institutional Ethics Committee of Animal Care and Use, Siriraj Hospital, Mahidol University, Thailand. The Institutional Review Committee approved the use of an animal model in this experimental protocol.

\section{Stem cell injection to guinea pig with spontaneously severe OA}

Each guinea pig with severe knee OA was anaesthetized initially with an intra-peritoneal injection of pentobarbital $(37 \mathrm{mg} / \mathrm{kg})$. MSC used in the experiment was determined for specific MSC characteristics as shown in Figure 1. A single injection of $1.5 \times 10^{6}$ MSC in $300 \mu \mathrm{L}$ NSS was injected into the joint space of the left knee of each animal, whereas, normal saline solution, as a control,

\section{Figure 1. MSC characteristics.}
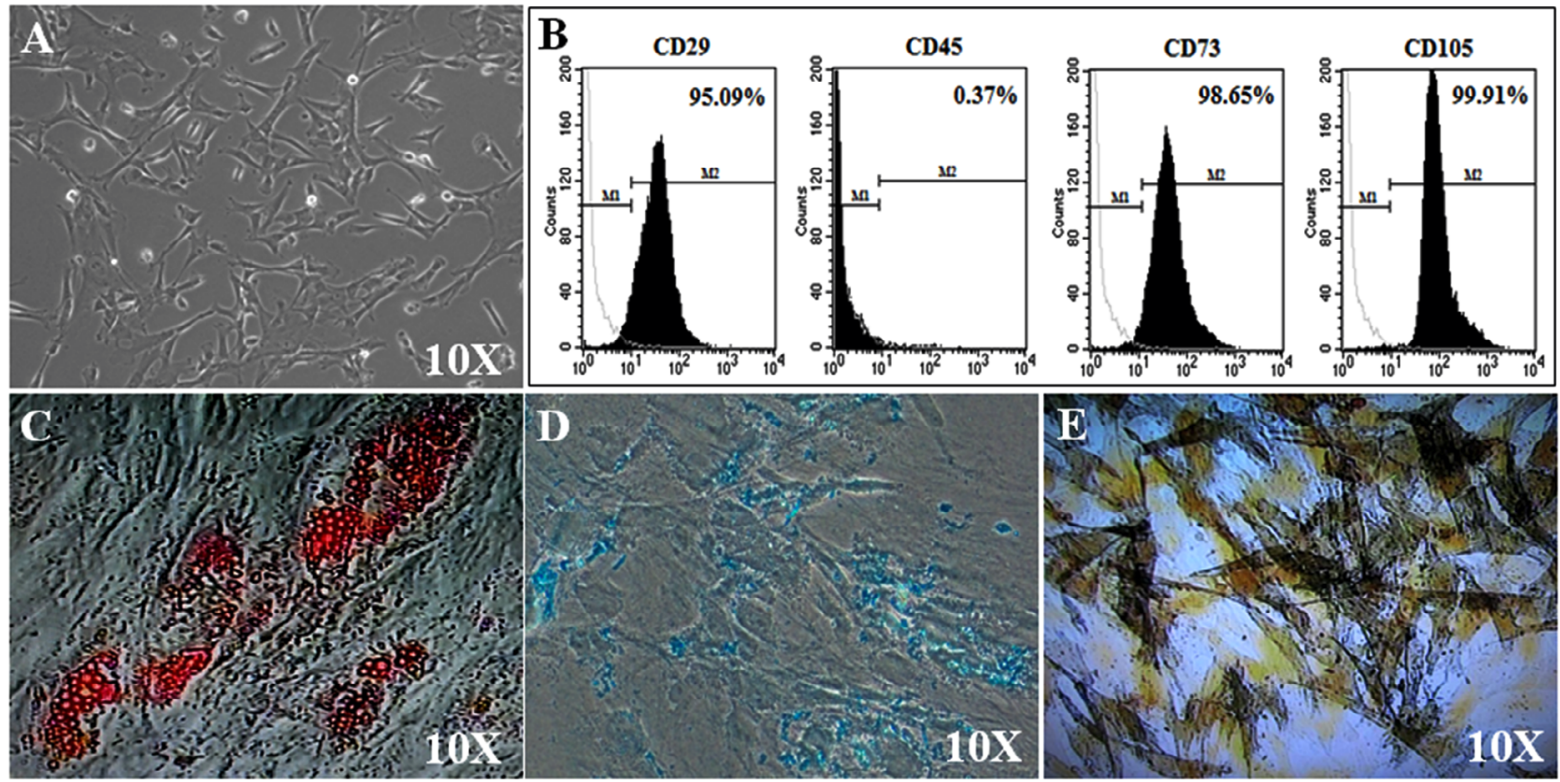

Morphologic appearance of MSC in phase-contrast images has been shown 10x magnification (A). Flow cytometer analysis reveals expression of antigenic phenotypic markers of CD29, CD73, CD105, but not CD45 on the MSC cell surface (B). MSC showed specific signals of stained lipid droplet for adipogenic lineage (in red) by Oil Red O staining (C), specific signals of alcian blue for chondrogenic lineage (in blue) (D), and appearance of osteogenic lineage (in brown) by alkaline phosphatase staining $(\mathrm{E})$ 
was injected into the joint space of right knee using a $30 \mathrm{G}$ needle.

\section{Evaluation of quality of life in OA animal model via ARom- De scores}

Gait Analysis: The hind-limb gait was measured with a footprint test of two hind-paws of the guinea pig [23]. The guinea pigs fasted for 6-8 $\mathrm{h}$ before the experiment. The hind-paws of the guinea pig were dipped in food coloring and placed at the beginning of the catwalk runway approximately $70 \mathrm{~cm}$ long. The guinea pig moved towards a food box at the end of the runway. The ink footprints were observed and a line was drawn perpendicular to the direction of the animal movement. The distance between footprints of each paw was measured and averaged to provide a gait length in each paw as shown in Figure 2A. The gait analysis was performed over seven periods of time, at $0,2,4,8,12,20$ and 28 weeks after the injections.

Weight Bearing: The guinea pigs fasted for 6-8 $\mathrm{h}$ before the experiment. The animals were placed on a runway of a jelly clay catwalk. As the guinea pig moved towards a food box at the end of the runway, the footprints of the hind-paws of guinea pig were imprinted in the clay. The evidence of foot-imprinting of hindpaws in the clay was determined by the volume of water in every step of the footprints of both paws. The volume of dropped water in each paw was averaged to provide the measure of weight bearing. The weight bearing on the left hind-limb is given as a percentage of total weight bearing on both hind-limbs in each animal. The percentage of weight bearing on investigating the hind-limbs was calculated with the formula of (volume of dropped water imprinted in clay of investigated hind-limb/volume of dropped water in imprinted clay of both hind-limbs) x 100 . The weight bearing was presented at grade scoring into a grade of 0,1 and 2 . The weight bearing analysis was performed for seven periods of time, at $0,2,4,8,12,20$ and 28 weeks after the injections.

Evaluation of overall ability of leg: To determine the overall ability of joint and muscle in OA knee guinea pig, the animal is wrapped into a vest supporting its chest, while both hind-limbs carried a weight of $100 \mathrm{~g}$. The guinea pigs were made to stand on two feet and lifted upward to the fixed bar. The animal was resting nicely with the hand supporting both hind-limbs until the experiment was started. In the experiment, both hind-limbs were allowed to lift a $100 \mathrm{~g}$ weight in a direction perpendicular to the ground as shown in Figure 2B. A number and a distance of a 100 $\mathrm{g}$ weight gain upward off the ground by leg lifting within two minutes were recorded and averaged to provide the overall ability of joint and muscle in each leg. The analysis was performed by the ratio of average lifting distance of left leg and the average lifting distance of the right leg in each animal. The overall ability of joint and muscle analysis presented as a grade scoring, into a grade of 0,1 and 2. The experiment was performed for seven periods of time, at $0,2,4,8,12,20$ and 28 weeks after the injections.

ARomDe scoring: In order to evaluate the quality of life in an old animal model with severe OA after MSC treatment, six parameters (including, gait analysis, weight bearing, the overall ability of leg, body weight, response to stimuli and grooming) were graded on a scale of 0 to 2 (Table 1 ). According to the importance of each parameter as they relate to physical recovery and mental behavior of an animal model, the sum of these six parameters was used to score the overall impact corresponding to quality of life using the ARomDe score. The scoring was graded from 0 to 12 thus: $(0-5=$ the treatment cannot affect an increase in quality of life, 6-9 $=$ the treatment can increase quality of life, 10-12 = the treatment has a high effect on the increase quality of life).

\section{Statistical analysis}

Results were presented as mean and standard error of the mean (mean \pm SEM). The gait analysis compared the distances among each hind-limb step of the same paws at different periods of study as compared to beginning of study at week 0 . The overall ability of each leg in an individual guinea pig was analyzed via the distances of $100 \mathrm{~g}$ weight lifting at different studied periods as compared to week two of the study. The statistical analysis of

Figure 2. Illustrations of the Methods.

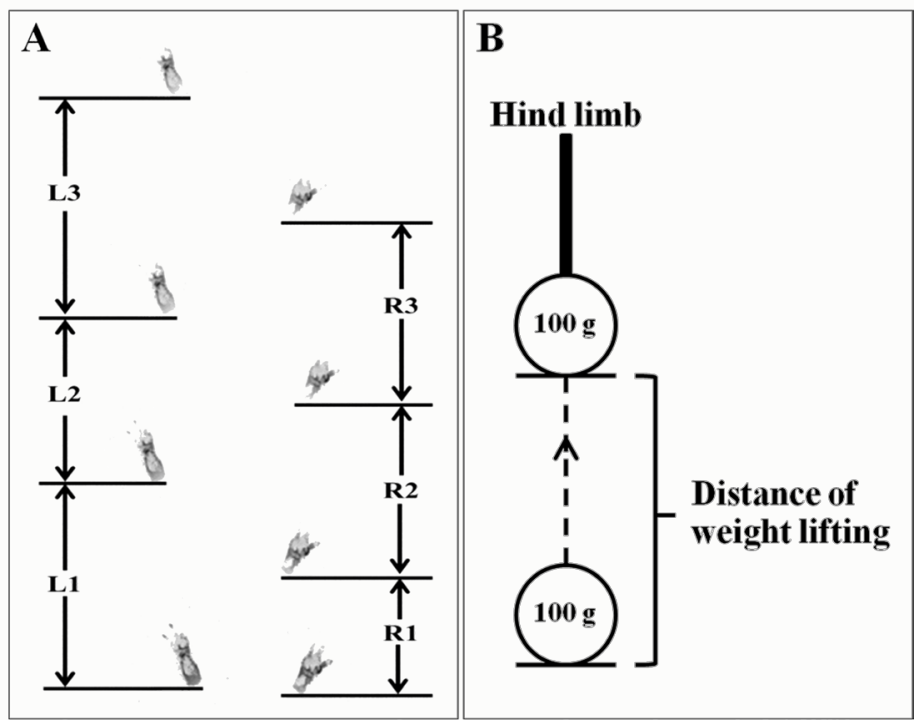

The gait length and the overall function of the leg for $100 \mathrm{~g}$ weight lifting were assessed in guinea pig. Gait length analysis is measured from footprints generated both hind-paws, which presented an average distance length of left paw ([L1+L2+L3]/3) and right paw ([R1+R2+R3]/3) (A). The overall function of the each leg for 100g weight lifting $(\mathrm{B})$ is measured from average distance of $100 \mathrm{~g}$ weight lifting in a direction perpendicular to the ground of left and right legs. 
Table 1. ARomDe Scoring scale.

\begin{tabular}{|c|c|c|}
\hline Parameter & Score & Score description \\
\hline \multirow{3}{*}{ Gait analysis } & 2 & increasing of distances between footprint step as compare to previous observation \\
\hline & 1 & no increasing of distances between footprint step as compare to previous observation \\
\hline & 0 & decreasing of distances between footprint step as compare to previous observation \\
\hline \multirow{3}{*}{ Weight bearing } & 2 & balance of weight bearing between two hind-limb \\
\hline & 1 & increasing of weight balance \\
\hline & 0 & no increasing of weight balance \\
\hline \multirow{3}{*}{ Overall ability of leg } & 2 & increasing leg ability of weight lifting \\
\hline & 1 & no increasing leg ability of weight lifting \\
\hline & 0 & decreasing leg ability of weight lifting \\
\hline \multirow{3}{*}{ Body weight } & 2 & body weight gain more than $5 \%$ \\
\hline & 1 & body weight did not change \\
\hline & 0 & body weight loss more than $5 \%$ \\
\hline \multirow{3}{*}{ Responding to stimuli } & 2 & active to surrounding environment \\
\hline & 1 & active when induce by stimuli \\
\hline & 0 & no activity, no interest to environment change \\
\hline \multirow{3}{*}{ Grooming } & 2 & self-grooming as usually, clean and clear fur \\
\hline & 1 & self-grooming for sometime \\
\hline & 0 & no self-grooming, messy and dirty fur \\
\hline
\end{tabular}

gait and overall ability and function was performed using student's paired t-test, GraphPad Prism 5 software (GraphPad, San Diego, CA). A value of $P<0.05$ was considered statistical significant.

\section{Results}

To provide an animal model which mimic elderly and obese patients with knee osteoarthritis in a severe stage, twenty white male Dunkin Hartley guinea pigs were fed and cared for a sterile environment with excess water, hay and pellets. One year old, guinea pigs were started the examination for their ability to walk on a runway once a week. After one and half years, most of them had stopped walking, and fifteen old guinea pigs were then subsequently naturally died. We observed the histology of knee cartilage of these fifteen guinea pigs by staining with Indian ink and found signs of severe osteoarthritis on the articular cartilage in both knees. At age two and half years, only five old guinea pigs, weighing 900-1400 g, met the requirements. They showed a disability to walk, which should be accompanied by the pain of naturally occurring severe $\mathrm{OA}$, ignoring to stimuli, no grooming, getting stress, and then could be used in the experiment.

These five surviving guinea pigs were MSC injected into intra articular of left knee joint and evaluated for quality of life by observation of pain, function recovery, behavior. Our results demonstrated that all animals had an increased quality of life and increasing the ability of daily activities, indicated by observation of pain reduction and recovery of knees and leg functions. Before the experiment started, all five guinea pigs looked lethargic and less motivated in the cage with no walking and no response to stimuli (such as water and food feeding, switch on-off the light and touching). They ignored self-grooming and change of environment. After two weeks of MSC intra-articular injection, three of the five guinea pigs started to move in short-step walks within the cage, whereas, two of five guinea pigs were not active. In week two, guinea pigs were determined fit for the walking test on the catwalk. We found that three of the five guinea pigs can continuously walk in two to five steps on a hard platform on the catwalk. The gait analysis was performed and showed that two of them have a longer distance between the footsteps of left paws than the distance of right paws. And one of them showed a comparable distance between the footsteps of left and right paws. At the four week observation, all five guinea pigs walked and moved within the cage. They became interested in their circumstances and responded to food feeding and mechanical stimuli and even self-grooming. During this week, all animals performed the walking tested on the $70 \mathrm{~cm}$ long catwalk. They were able to non-stop walk to the end of the runway. The gait analysis indicated the significant progression $(\mathrm{P}<0.05)$ of functional recovery and pain relief as observed at the increasing number of footsteps and distances between footstep of left and right paws. The guinea pigs can walk rapidly, continuously and enthusiastically, which was different from the time before MSC treatment. The functional recovery of guinea pigs showed a subsequently progressive recovery further into week 20 of the study. After 20 weeks of MSC injection to the knee joint, we found a reduction of step distance by gait analysis; however, all animals can still continue a non-stop walking on the long catwalk until the end of the runway. The data of guinea pig No.2 can be recorded only 27 weeks due to natural death from old of the animal during the experiment. The result of gait analysis has been shown in Figure 3A.

For weight bearing analysis, the experiment was performed and determined via the volume of foot-imprints of right and left paws in the jelly clay. The guinea pigs were placed and walked on the jelly clay platform on the catwalk. They left the imprints of footsteps on the clay, which allowed us to measure the weight bearing of paws by volume of water.

At week two of our study, all guinea pigs had detected a bias of 
Figure 3. Analysis of Gait and weight bearing in OA animal model.

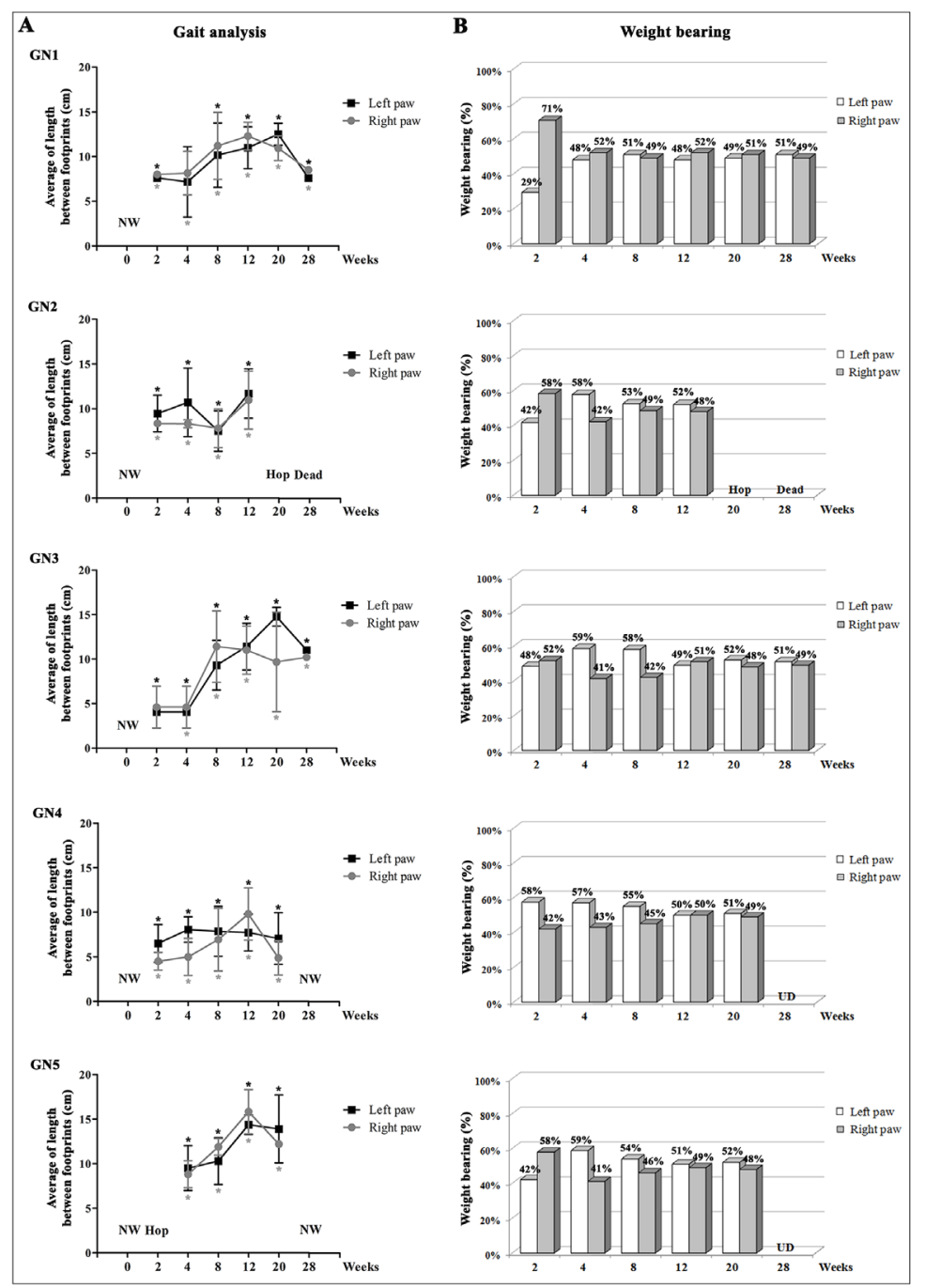

(A) Gait analysis indicates distance between steps of footprints. All data were shown as means $\pm \mathrm{SEM}$ (P-value $<0.05$ ). The superscript $*$ refers to a statistically significant difference as compared to the beginning of the study in week $0 . \mathrm{NW}=$ No walk, Hop $=$ Hopping. (B) The data were presented in percentage of weight bearing in left and right paws of animal model at each period of experiment. The result showed a balance of weight bearing after eight weeks to the end of the study. UD = undetected, Hop = Hopping.

weight-bearing between left and right hind-limbs. After three weeks of MSC injection in left OA joints, all five animals showed subsequent increase of the weigh-bias in the left hind-limbs, indicating the recovery progression of left knee joint. Interestingly, we found that every guinea pig achieved a balance of weight bearing between left and right knees after four weeks of injections. Moreover, they could walk continuously and nimbly on hard platform catwalk. The balance of weight bearing of left and right hind-limbs was continuous until the end of the study. The result of weight-bearing analysis has been shown in Figure 3B.

For assessment of the overall ability of their legs, we set up the guinea pigs to vertically lift the $100 \mathrm{~g}$ weight and observed the ability of using their leg and muscle strength. The result showed that the left leg, which was MSC transplanted into the knee joint, showed superiority of ability and function to the right leg that was the control. From the above finding, it is clear that the treatment with a MSC intra-articular injection can increase the quality of life in old animal model with severe OA knee by pain relief and achieve the recovery of function and ability of daily activities. The functional recovery of the overall ability of the leg has been shown in Figure 4.
For evaluation of the quality of life via the ARomDe score, six parameters including, gait analysis, weight bearing, the overall ability of leg, body weight, responding to stimuli and grooming, were scored (Table 2) and plotted in the graph (Figure 5). After MSC injection in week two, the plotting graph of ARomDe scores demonstrated the ascending curve in all five guinea pigs. The score was found to increase and be maintained to week 20 . After week 24 of the study, the plotting graph of every animal model started a descending curve of score plotting. However, after week 28 of the study, the plotting score of ARomDe scoring retained at a higher level as compared to the ARomDe score at the beginning of the study. This finding showed a consistency of results in every animal model. The result of ARomDe scores, indicating quality of life, has been shown in Figure 5.

\section{Discussion}

This study investigated the effect of OA treatment with a simple intra-articular injection of $1.5 \times 10^{6}$ human MSC in an old animal model with naturally severe $\mathrm{OA}$ and obesity to monitor the qual- 
Figure 4. Progressive pattern of the overall ability of each old animal model with severe natural OA knee.

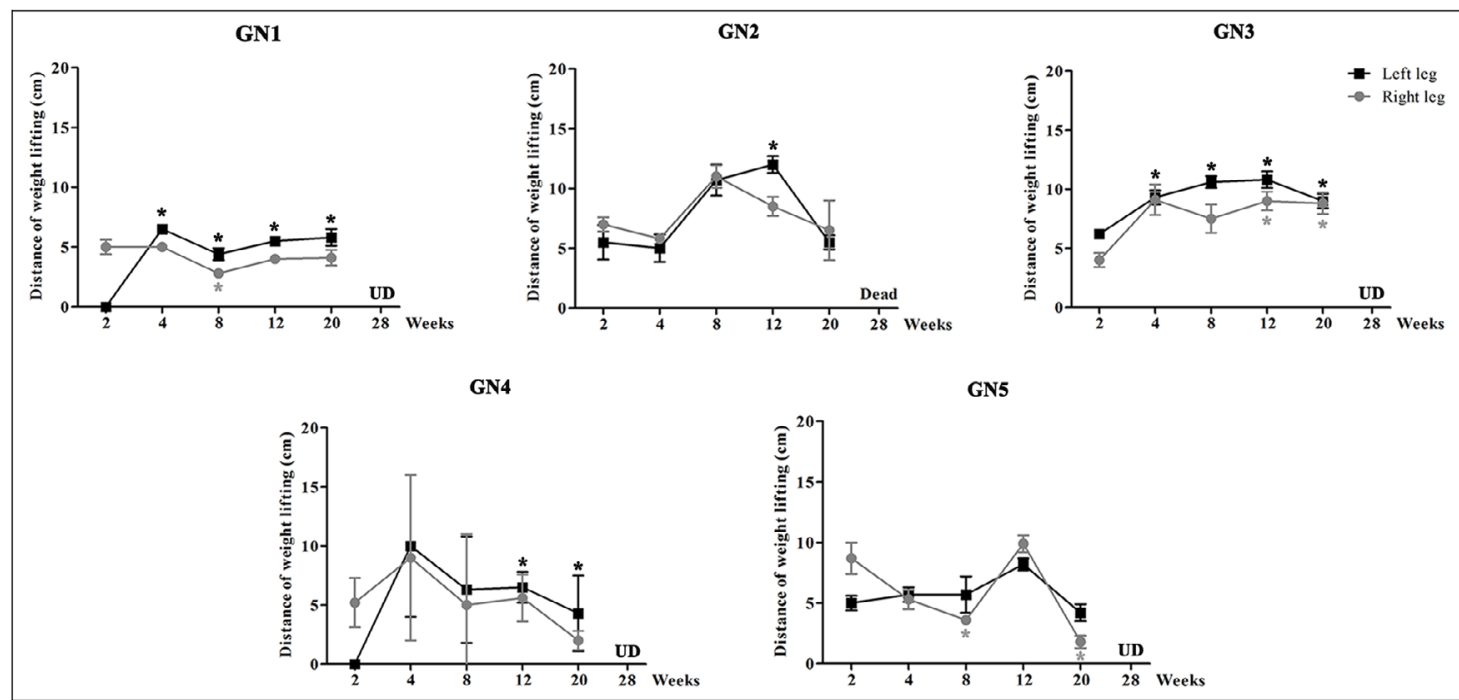

The study of the ability of leg function in an animal model was started in week two after MSC treatment. All animals displayed a subsequent progression of overall ability of leg function after therapy as observed from their ability for vertical lifting of $100 \mathrm{~g}$ weigh. Data are presented as means \pm SEM. The superscript $*$ refers to significant differences $(\mathrm{P}$-value $<0.05)$ of each data as compared with data in week two of the study within each leg. UD $=$ undetectable. Data of guinea pig number two at week 28 was not obtained because of the natural death of the guinea pig.

Table 2. Six parameters of ARomDe scoring test in individual animal model.

\begin{tabular}{|c|c|c|c|c|c|c|c|}
\hline \multirow[t]{2}{*}{ Parameters } & \multirow[t]{2}{*}{ Guinea pig number } & \multicolumn{6}{|c|}{$\begin{array}{l}\text { After stem cell injection } \\
\text { (weeks) }\end{array}$} \\
\hline & & 2 & 4 & 8 & 12 & 20 & 28 \\
\hline \multirow{5}{*}{ Gait analysis } & GN1 & 1 & 2 & 2 & 2 & 2 & 0 \\
\hline & GN2 & 1 & 2 & 0 & 2 & 0 & dead \\
\hline & GN3 & 1 & 2 & 2 & 2 & 2 & 2 \\
\hline & GN4 & 1 & 2 & 2 & 2 & 2 & 2 \\
\hline & GN5 & 1 & 2 & 2 & 2 & 2 & 2 \\
\hline \multirow{5}{*}{ Weight bearing } & GN1 & 0 & 2 & 2 & 2 & 2 & 2 \\
\hline & GN2 & 0 & 1 & 2 & 2 & 0 & dead \\
\hline & GN3 & 0 & 1 & 2 & 2 & 2 & 2 \\
\hline & GN4 & 0 & 0 & 2 & 2 & 2 & 2 \\
\hline & GN5 & 0 & 1 & 2 & 2 & 2 & 2 \\
\hline \multirow{5}{*}{ Overall ability of leg } & GN1 & 1 & 2 & 2 & 2 & 2 & 0 \\
\hline & GN2 & 1 & 0 & 2 & 2 & 2 & dead \\
\hline & GN3 & 1 & 2 & 2 & 2 & 2 & 0 \\
\hline & GN4 & 1 & 2 & 2 & 2 & 2 & 0 \\
\hline & GN5 & 1 & 2 & 2 & 2 & 0 & 0 \\
\hline \multirow{5}{*}{ Body weight } & GN1 & 1 & 1 & 1 & 2 & 1 & 1 \\
\hline & GN2 & 1 & 1 & 1 & 1 & 2 & dead \\
\hline & GN3 & 1 & 0 & 1 & 1 & 1 & 1 \\
\hline & GN4 & 1 & 1 & 1 & 1 & 2 & 1 \\
\hline & GN5 & 1 & 0 & 1 & 0 & 2 & 2 \\
\hline \multirow{5}{*}{ Responding of stimuli } & GN1 & 2 & 2 & 2 & 2 & 2 & 2 \\
\hline & GN2 & 0 & 1 & 1 & 2 & 0 & $\overline{\text { dead }}$ \\
\hline & GN3 & 0 & 1 & 1 & 1 & 2 & 1 \\
\hline & GN4 & 0 & 0 & 1 & 1 & 0 & 1 \\
\hline & GN5 & 2 & 2 & 1 & 1 & 2 & 0 \\
\hline \multirow{5}{*}{ Grooming } & GN1 & 2 & 2 & 2 & 1 & 1 & 2 \\
\hline & GN2 & 2 & 1 & 2 & 2 & 2 & dead \\
\hline & GN3 & 1 & 1 & 2 & 0 & 0 & 1 \\
\hline & GN4 & 0 & 2 & 2 & 1 & 1 & 0 \\
\hline & GN5 & 1 & 2 & 1 & 1 & 1 & 1 \\
\hline
\end{tabular}


Figure 5. Plotting curve of ARomDe score.

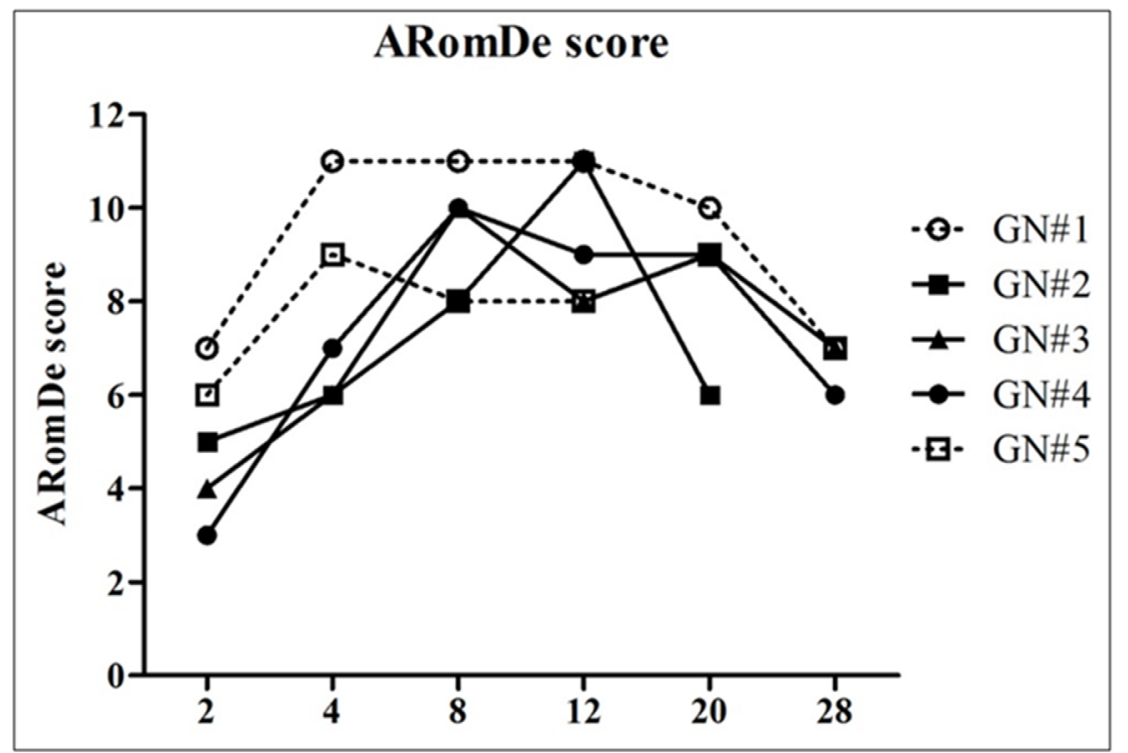

The ARomDe scores were calculated by the sum of six parameters identified for pain relief, recovery of physical function, mental and behavior, and the scores of each guinea pig were plotted into the graph. The graph showed a consistent pattern, indicating the increasing quality of life in all guinea pigs after the treatment of MSC therapy with a single injection to intra-articular.

ity of life in guinea pig models. The quality of life was evaluated using the ARomDe score via three dimensions, which indicated pain, physical recovery and behavior, by six analysis parameters, including gait analysis, weight bearing, the overall ability of leg, body weight, responding to stimuli, and grooming. The progression of the overall well-being in six studied parameters was documented in all guinea pigs as could recover the daily activities, such as interesting in appetite, grooming and moving within the cage. Surprisingly, the recovery of the knee joint after MSC transplantation was not only found on the MSC-injected knee joint, the recovery was also subsequently found in their walking function. The recovery of function was observed at week four of the study as identified by a walking test on hard runway.

Although, the improvement can be found in both knee joints (MSC-injected knee joint and control knee joint) of each animal, the left knee joint which was transplanted with MSC therapy showed superior characters of function recovered as observed in a longer distance in footprint steps using gait analysis and a greater ability of weight lifting in leg function experiment. However, our work found that the recovery could be retained for a longer period of time. The progression is subsequently increased up to week four of the study and can be maintained for a period and gradually decreased at six months after MSC injection. This implied the need of a repeated MSC injection after six months.

MSC transplantation has been studied in various animal models and achieved the success, such as significantly improvement of joint function in equine [19], improving mobility of dog's OA joint [20], inducing reparation of knee cartilage [22]. Our work showed consistent results of improvement of OA joint and function recovery in animal models.

The Dunkin Hartley guinea pigs have been used as animal models for osteoarthritis in our experiment and previous reports due to their spontaneously developed degenerative cartilage changes mimic those of human and are similar to those occurring in the most common forms of slowly progressive human disease [24, 25]. Occurrence of severe $\mathrm{OA}$ in guinea pig has been reported at 18 months to two years old [24]. To obtain the animal model that meet the criteria, we housed and cared for guinea pigs until they reached 2.5 years of age, weighed more than $900 \mathrm{~g}$ and had stopped walking due to OA pain to meet the criteria of advanced age, obesity and spontaneous severe OA of the knee, as a representative of elderly populations with obesity and severe OA. However, this study started with 20 animals initially, but only five animals completed the experiment due to the progression of age and ultimately death.

According to the study performed in this animal model, which is age advanced and experiencing pain caused by severe $\mathrm{OA}$, the animals easily found stress in an unfamiliar environment and complicated equipment, resulting in stopped actions of the animal during the experiment. In order to provide suitable parameters for evaluation of the quality of life in an old guinea pig with severe OA, we set up the "ARomDe score" measurement system for the first time. The ARomDe score monitors the quality of life via the measurement of three dimensions, including pain and function recovery and behavior. It is consistent with many previous studies, which generally evaluate the improvement of models and human with knee osteoarthritis via the achievement of pain relief [2, $7,14,19,20,26,28,29]$ and function recovery $[1,2,26,27]$ and emotional behavior $[1,19,20,27]$.

Three main dimensions of the ARomDe score (analysis of pain and daily function and emotional behavior) were assessed by six parameters (gait analysis, weight bearing, the overall ability of leg function, body weight, responsiveness to stimuli and grooming). The parameters for pain behavior of arthritic animals measure sensitivity of knee joint of the hind-paws. Indirect measures (gait analysis and weight bearing) of knee joint pain generally used to assess pain in various animal models and even in human [30-34]. For function recovery overall of the leg, we attended to the main challenge of function, ability and mechanical strength, and then 
developed them as a key parameter in the ARomDe score. OAinduced pain can affect behavior alterations in OA models. Thus, changed behavioral parameters can be used in interpreting knee OA-induced pain response. Grooming and responding to stimuli are behavioral parameters [35], which are mainly used for behavioral analysis. ARomDe score analyzed behavior via the parameter of grooming and responsiveness of animal model to stimuli, which motivated with an attached $100 \mathrm{~g}$ weight.

The high prevalence of OA disease in the elderly population is increasing year by year in the world population. Surgical complication risks for knee arthroplasties were found to increase starting at age 70 years with an average range from $4 \%$ for $40-64$ years old to $17 \%$ in 90 years old [36]. Most old patients often refuse the total knee surgery. Attempting to provide a new simple treatment of MSC therapy is needed. Although, stem cell therapy in OA knee has been studied in many previous works, most of them focus their attention on the neo-cartilage tissue regeneration $[12,14,22$, $37,38]$, which may not be a main interest in older patients. In contrast, stem cell therapy based on quality of life instead of tissue regeneration would become advantageous and a major interest of OA treatment in older patients. Our work contributes to the in vivo study to determine the quality of life in this aged advanced animal model for mimicking the OA elderly population, who suffers with great pain, disability in daily activities and hopelessness with conservative treatment. Guinea pigs used in this experiment were getting very old and met the criteria with signs of $O A$ pain, loss of daily walking, activities and social interaction and ignoring the surrounding environment and attempting to groom themselves. Moreover, this study performed the monitoring of the quality of life in OA aged animals using a series of scientific parameters for evaluating of pain, function and emotional behavior.

Our work demonstrates novelty evidence that a single injection of MSC into intra-articular cartilage can increase the quality of life in an old model with severe OA suffering by clearing pain and recovery of leg function and daily activities. It can then imply that a single intra-articular injection of the MSC can be a further routine treatment of $\mathrm{OA}$ in aged people.

\section{Acknowledgement}

This work was supported by Siriraj Foundation, (Funds number: D003631), Siriraj Hospital, Thailand and Wincell Co.Ltd granted to Dr. Tatsanee Phermthai. The authors are grateful to Leslee Sinclair and Peter A. McGuin for manuscript editing.

\section{References}

[1]. Farr II, Miller LE, Block JE (2013) Quality of life in patients with knee osteoarthritis: a commentary on nonsurgical and surgical treatments. Open Orthop J. 7: 619-623.

[2]. Kawano MM, Araujo IL, Castro MC, Matos MA (2015) Assessment of quality of life in patients with knee osteoarthritis. Acta Ortop Bras. 23(6): 307-310.

[3]. Ferrell BA (1991) Pain management in elderly people. J Am Geriatr Soc. 39(1): 64-73.

[4]. Woolf AD, Pfleger B (2003) Burden of major musculoskeletal conditions. Bull World Health Organ. 81(9): 646-656.

[5]. Berger MJ, Kean CO, Goela A, Doherty TJ (2012) Disease severity and knee extensor force in knee osteoarthritis: data from the Osteoarthritis Initiative. Arthritis Care Res. 64(5): 729-734.

[6]. Kon E, Filardo G, Drobnic M, Madry H, Jelic M, et al., (2012) Non-surgical management of early knee osteoarthritis. Knee Surg Sports Traumatol Ar- throsc. 20(3): 436-449.

[7]. Wiegant K, Van Roermund PM, Intema F, Cotofana S, Eckstein F, et al., (2013) Sustained clinical and structural benefit after joint distraction in the treatment of severe knee osteoarthritis. Osteoarthritis Cartilage. 21(11): $1660-1667$.

[8]. Kuo FC, Hsu CH, Chen WS, Wang JW (2014) Total knee arthroplasty in carefully selected patients aged 80 years or older. J Orthop Surg Res. 9: 61.

[9]. Liu J, Yuan W, Wang X, Royse CF, Gong M, et al., (2014) Peripheral nerve blocks versus general anesthesia for total knee replacement in elderly patients on the postoperative quality of recovery. Clin Interventions Aging. 9: 341350.

[10]. Robinson TN, Raeburn CD, Tran ZV, Angles EM, Brenner LA, et al., (2009) Postoperative delirium in the elderly: risk factors and outcomes. Ann Surg. 249(1): 173-178.

[11]. Pedersen AB, Mehnert F, Johnsen SP, Husted S, Sorensen HT (2011) Venous thromboembolism in patients having knee replacement and receiving thromboprophylaxis: a danish population-based follow-up study. J Bone Joint Surg Am. 93(14): 1281-1287.

[12]. Koh YG, Choi YJ, Kwon SK, Kim YS, Yeo JE (2015) Clinical results and second-look arthroscopic findings after treatment with adipose-derived stem cells for knee osteoarthritis. Knee Surg Sports Traumatol Arthrosc. 23(5): 1308-1316.

[13]. Jo CH, Lee YG, Shin WH, Kim H, Chai JW, et al., (2014) Intra-articular injection of mesenchymal stem cells for the treatment of osteoarthritis of the knee: a proof-of-concept clinical trial. Stem cells 32(5): 1254-1266.

[14]. Soler R, Orozco L, Munar A, Huguet M, Lopez R, et al., (2016) Final results of a phase I-II trial using ex vivo expanded autologous mesenchymal stromal cells for the treatment of osteoarthritis of the knee confirming safety and suggesting cartilage regeneration. Knee. 23(4): 647-654.

[15]. Koelling S, Miosge N (2009) Stem cell therapy for cartilage regeneration in osteoarthritis. Expert Opin Bio Ther. 9(11): 1399-1405.

[16]. Nöth U, Steinert AF, Tuan RS (2008) Technology insight: adult mesenchymal stem cells for osteoarthritis therapy. Nat Clin Pract Rheumatol. 4(7): 371-380.

[17]. Davatchi F, Abdollahi BS, Mohyeddin M, Shahram F, Nikbin B (2011) Mesenchymal stem cell therapy for knee osteoarthritis. Preliminary report of four patients. Int J Rheum Dis. 14(2): 211-215.

[18]. Vinatier C, Bouffi C, Merceron C, Gordeladze J, Brondello JM, et al., (2009) Cartilage tissue engineering: towards a biomaterial-assisted mesenchymal stem cell therapy. Curr Stem Cell Res Ther. 4(4): 318-329.

[19]. Broeckx S, Zimmerman M, Crocetti S, Suls M, Marien T, et al., (2014) Regenerative therapies for equine degenerative joint disease: a preliminary study. PLoS One. 9(1): e85917.

[20]. Tsai SY, Huang YC, Chueh LL, Yeh LS, Lin CS (2014) Intra-articular transplantation of porcine adipose-derived stem cells for the treatment of canine osteoarthritis: A pilot study. World J Transplant. 4(3): 196-205.

[21]. Jimenez PA, Glasson SS, Trubetskoy OV, Haimes HB (1997) Spontaneous osteoarthritis in Dunkin Hartley guinea pigs: histologic, radiologic, and biochemical changes. Lab Anim Sci. 47(6): 598-601.

[22]. Sato M, Uchida K, Nakajima H, Miyazaki T, Guerrero AR, et al., (2012) Direct transplantation of mesenchymal stem cells into the knee joints of Hartley strain guinea pigs with spontaneous osteoarthritis. Arthritis Res Ther. 14(1): R31.

[23]. Steinberg H, Sykes EA, McBride A, Terry P, Robinson K, et al., (1989) Computer analysis, using a digitizer, of ataxic mouse gait due to drugs. J Pharmacol Methods. 21(2): 103-113.

[24]. Bendele AM (2001) Animal models of osteoarthritis. J Musculoskelet Neuronal Interact. 1(4): 363-376.

[25]. Young DM, Fetter AW, Johnson LC (1979) Spontaneous animal models of human disease (1st Edn), volume 2. Academic Press, Inc., New York.

[26]. Vonsy JL, Ghandehari J, Dickenson AH (2009) Differential analgesic effects of morphine and gabapentin on behavioural measures of pain and disability in a model of osteoarthritis pain in rats. Eur J Pain. 13(8): 786-793.

[27]. Schneider T, Welker P, Licha K, Haag R, Schulze-Tanzil G (2015) Influence of dendritic polyglycerol sulfates on knee osteoarthritis: an experimental study in the rat osteoarthritis model. BMC Musculoskeletal Disord. 16: 387.

[28]. Khunakornvichaya A, Lekmeechai S, Pham PP, Himakoun W, Pitaksuteepong T, et al., (2016) Morus alba L. stem extract attenuates pain and articular cartilage damage in the anterior cruciate ligament transection-induced rat model of osteoarthritis. Pharmacology. 98(5-6): 209-216.

[29]. Fernihough J, Gentry C, Malcangio M, Fox A, Rediske J, et al., (2004) Pain related behaviour in two models of osteoarthritis in the rat knee. Pain. 112(1): 83-93.

[30]. Otsuki T, Nakahama H, Niizuma H, Suzuki J (1986) Evaluation of the analgesic effects of capsaicin using a new rat model for tonic pain. Brain Res. 365(2): 235-240.

[31]. Schött E, Berge OG, Ängeby-Möller K, Hammarström G, Dalsgaard CJ, et 
al., (1994) Weight bearing as an objective measure of arthritic pain in the rat. J Pharmacol Toxicol Methods. 31(2): 79-83.

[32]. McDougall JJ, Watkins L, Li Z (2006) Vasoactive intestinal peptide (VIP) is a modulator of joint pain in a rat model of osteoarthritis. Pain. 123(1): 98-105.

[33]. Combe R, Bramwell S, Field MJ (2004) The monosodium iodoacetate model of osteoarthritis: a model of chronic nociceptive pain in rats? Neurosci Lett. 370(2): 236-240.

[34]. Clarke KA, Heitmeyer SA, Smith AG, Taiwo YO (1997) Gait analysis in a rat model of osteoarthrosis. Physiol Behav. 62(5): 951-954.

[35]. Ellen Y, Flecknell P, Leach M (2016) Evaluation of using behavioural changes to assess post-operative pain in the guinea pig (Cavia porcellus). PloS One. 11(9): e0161941.

[36]. Easterlin MC, Chang DG, Talamini M, Chang DC (2013) Older age increases short-term surgical complications after primary knee arthroplasty. Clin Orthop Relat Res. 471(8): 2611-2620.

[37]. de Windt TS, Vonk LA, Slaper-Cortenbach I, van den Broek MP, Nizak R, et al., (2017) Allogeneic mesenchymal stem cells stimulate cartilage regen- eration and are safe for single-stage cartilage repair in humans upon mixture with recycled autologous chondrons. Stem cells. 35(1): 256-264.

[38]. Desando G, Giavaresi G, Cavallo C, Bartolotti I, Sartoni F, et al., (2016) Autologous bone marrow concentrate in a sheep model of osteoarthritis: new perspectives for cartilage and meniscus repair. Tissue Eng Part C Methods. 22(6): 608-619.

\section{Special Issue on}

"Human Stem cells \& Transplantation"

Edited by:

Guanghui Liu, Chinese Academy of Sciences, China.

E-mail: ghliu@ibp.ac.cn 